\title{
Current Research Trends in Modification / Interaction of Halloysite Nanotube Filled Polymer Blends and Its Composites: A Review
}

\author{
Pravin Kubade ${ }^{1}$, Ravindranath Kshirsagar ${ }^{2}$ \\ ${ }^{1}$ SMBS, VIT University, Vellore-632 014, TN, India \\ ${ }^{2}$ TKIET college of Engineering, Warnanagar, Kolhapur, India
}

\begin{abstract}
These Halloysite is a naturally occurring aluminosilicate material (Al2Si2O5(OH)4.2H2O) with hollow tubular structure which is obtained from mines as natural deposits. Halloysite shows unique surface chemical property because of its multi-layer structure with a few hydroxyl groups present on the surface of the nanotubes. Due to the weak secondary interactions between the nanotubes by hydrogen bonds and van derWaal's forces halloysite can be easily dispersed in a non-polar polymer matrix such as polupropylene as compared to any other layer silicates. Today we know the HNT's filled polymer blends and its composites is because of various researches carried out over many years. Researchers have toiled hard to improve the thermomechanical performance and physical properties with various experiments. A looking down with hatred of different approaches, every new research shares the same objectives of getting superior thermomechanical performance and physical properties at very lower filler loadings compared to conventional polymer composites. This paper reviews the vast array of research work carried out within past decades for the modifications/intercalations of HNTs to improve the dispersion and physical properties of the nanocomposites. This study is mainly focused on the aspects related to improve the mechanical, thermal and physical properties of the nanocomposite. It reports the research trends in modification/interaction of HNTs filled polymer blends and its composites.
\end{abstract}

Keywords: HNTs, aluminosilicate (A12Si2O5(OH)4.2H2O).

\section{Introduction}

Polymer nanocomposites are new category of materials which are filled with nanofillers, and which usually shows exceptionally superior thermomechanical performance and physical properties as compared to conventional polymer composites. Halloysite nanotubes are readily available, they are not only cheaper than other nanofillers such as carbon nanotubes but also they are biocompatible. Polypropylene doesn't have any polar groups to interact with the halloysites. This generally results in poor dispersion of the halloysites in the PP matrix and also the poor interfacial bonding between the filler surface and the PP matrix. This also limits the advantages of incorporation of the halloysites into the PP matrix. Due to its multi-purpose features the naturally occurring tubular halloysite clay has attracted many interest of the researchers. This nanomaterials can be used as nanofiller for polymers. Reinforcement of thermoplastics matrices with HNTs has been studied enormously by different research categories. HNTs can used in polymeric nanocomposites to improve the mechanical strength, thermal stability, crystalline behavior etc. of the polymers [1-2]. In determining the final performance of polymer nanocomposites the interfacial interactions and the degree of dispersion of fillers in polymer matrix are key issues [10]. Chemically, HNTs are acknowledged for their relatively lower hydroxyl density on their outer surfaces compared with fumed silica and other layered silicates such as montmorillonite [20]. The aggregation prevailed upon by the intertubular hydrogen bonding is susceptible to the shearing force. In true incident, morphology study for many polymer/HNTs composites has shown single-tube dispersed halloysites in the matrix [23]. More recently, some studies have being reported the effects of HNT loading (wt. \%) and surface modification on the flame retardant property and thermal stability of PP/HNTs nanocomposites [4]. Furthermore, surface modification or treatment of the fillers also imparts good wetting property of with polymers by adding nanoparticles in a solution process [5-6]. Due to its well-balanced physical and mechanical properties and easy processability at a relatively low cost Polypropylene (PP) as an important commercial plastic is widely used to produce household goods and automotive parts. The application of PP in various fields such as industrial, medical and other fields or sectors can be further expanded once its mechanical performances have been highly upgraded. Therefore, PP has been a popular matrix used in unit with all types of nanofillers. Wt. \% loading of HNT and its surface treatment/modification plays vital role in enhancing the dispersion rate, thermomechanical and physical properties of nanocomposites $[4,15,23,24]$. The interfacial interactions between HNTs and SBR through SA improves by SA as an interfacial modifier for SBR/HNTs nanocomposite. The largely improved interfacial bonding between HNTs, rubber and significantly enhanced dispersion of HNTs were correlated with the significantly improved mechanical properties [7, 20] were documented. Halloysite modification/treatment by hexa-decyl-tri-methyl-ammoniumbromide,3-aminopropyltrimethoxysilane and the urea individually and also in combine using in situ modification/treatment of the PP matrix by 4,4!diphenylmethylene dimaleinimide (DBMI) [25] is also reported in this study. However, an organic-inorganic film based on the HNTs and chitosan was examined throughly as animmobilization matrix to entrap HRP, where the chitosan can be used as binder binder to enhance the adhesiveness of 


\section{International Journal of Science and Research (IJSR) \\ ISSN (Online): 2319-7064 \\ Index Copernicus Value (2013): 6.14 | Impact Factor (2014): 5.611}

the composite film to the electrode surface. The nanocomposite film gives favorable micro-environment for HRP to perform DET as well as it retain its bioactivity, was also reported. The nanocomposite film obtained because of electrocatalytic reduction of $\mathrm{H} 2 \mathrm{O} 2$ at $\mathrm{HRP} / \mathrm{HNTs} / \mathrm{Chi}$ nanocomposite film-modified electrodes is attractive for both electrochemical investigations of heme proteins as well as for the development of an amperometric biosensor [13]. The curing characteristics, morphology and the mechanical properties of HNT nanocomposite are affected by the interfacial hydrogen bonding [3] At present, few approaches have been reported about preparing HNTs/PPy coaxial tubular nanocomposite where low-costing natural halloysite nanotubes were used to produce nanocomposites with welldefined coaxial tubular morphology for the first time. Furthermore, because of their insulating property halloysite nanotubes can be used as a support for the polypyrrole coatings have being reported [8]. With an electroless plating the continuous deposition of $\mathrm{Ni}$ nanoparticles on the outer surface and wires in the cavity of the halloysite template is obtained. It is necessary to activate the halloysite template in order to perform the electroless plating. The catalyst as a colloidal Sn-Pd solution requires a complicated treatment its strong acidity destroy clay substrate also. However, in order to initiate the electroless deposition of nickel the in situ reduction of $\mathrm{Pd}$ ions by a methanol on surface of halloysite can be conducted. Furthermore, the investigation has been done regarding the magnetic property of as-plated or the post-annealed $\mathrm{Ni}$ on template when template is oriented in the resin and aligned in order to subject the magnetic field [19].

Melt mixing is a most advisable method because it combines high shear with simplicity. Furthermore, it doesn't have any solvents, contaminants and also equipment required for efficient production of large quantity of nanocomposites is already exists in the plastics industry. In all melt mixing techniques, for the successful preparation of nanocomposites good interfacial adhesion and a homogeneous dispersion are crucial. The response to properties such as thermomechanical, morphological, fire retardant and water vapor permeability are determined by processing and varying the concentrations of HNTs filled polymers and its composites [14-16-17-18].

One of the most promising nanocomposite are the rubber/clay nanocomposites. The addition of halloysite nanotubes (HNTs) into EPDM enhances the thermomechanical properties has been reported [12]. In fact EPDM and HNTs are incompatible because EPDM doesn't contain any polar groups. Also it has been reported that incorporation of the maleic anhydride via peroxide-initiated melt grafting onto EPDM and its use as a compatibilizer in EPDM/HNT nanocomposites, which shown higher tensile properties, further the comparison between the tensile properties and morphological characteristics of uncompatibilized and compatibilized EPDM/HNT nanocomposites propose a reinforcement mechanism which shows the interactions inside the EPDM/ MAH-gEPDM/HNT nanocomposites [11]. The environmentally friendly HNTs as fillers in thermoplastics such as polyamide66 (PA66), biobased polyamide 11(PA11), polypropylene
(PP), and thermoplastic starch (TPS) matrices to generate new kind of nanocomposite by advantageous melt-processing method have been presented by author. The effects of HNTs loading on various properties such as optical, thermomechanical, structural, viscoelastic and dielectic properties were investigated. Consequently, it has been concluded that HNTs are most promising, green and unique reinforcing material for thermoplastics [22].

The crystallization behaviors study including polymorphism and non-isothermal crystallization kinetics of PA6/HNTs nanocomposites was also reported. Where the Mo and empirical kinetics equations as Avrami equation equation are used to analyze the temperature and crystallization rate of nanocomposites on the HNTs Wt. \% as well as cooling rate and its dependency. In addition with this, WXRD was used to detect the dependency of crystallinity as well as polymorphism of nanocomposites on cooling rate and HNTs wt. $\%$ addition while non-isothermal process of crystallization and then the crystallization behavior was correlated with HNTs and PA6 chain interaction [21]. In this review paper, various trends in HNT filled polymer blends and its composites and associated thermomechanical performance and physical properties has been considered involving effect of HNT loading wt. \%, surface modification/treatment of HNT, EDPM nanocomposites filled by halloysite, dispersion of HNTs, HNT modification, nanocomposites based on silicate nanotubes, morphology and crystallization behavior, and environmentally friendly HNTs.

\section{Effect of HNT Loading (wt. \%)}

H. Ismail and S.M. Shaari [1] revealed that increase of HNTs in the weight ratio of $\mathrm{PA} / \mathrm{HNT}$ s shows an increasing trend in terms of cure time, scorch time, maximum torque, tensile strength and tensile modulus (M100 and M300) of PA/HNTs/EPDM hybrid composites but reduces the elongation at break. By the rubber-filler interactions measurements and SEM study author concluded that HNTs has better adhesion to EPDM matrix than the palm ash.

The influence of molar mass of polyamide 6 on the mechanical, thermal and rheological properties of PA 6/halloysite nanocomposites was studied by Ulrich A. Handge et all. [14]. They stated that, with increase in HNTs modulus as well as yield stress of polyamide increases, an effect which is very much distinct at low filler fractions for the low molar mass PA 6 . The storage modulus and modulus of elasticity linearly increase with concentration of HNTs, at large filler fraction. With $30 \mathrm{wt}$. \% addition of HNTs the Young's modulus increases by $90 \%$. It was also observed that above the glass transition temperature $(\mathrm{Tg})$ of PA 6 the reinforcement effect of HNT's is very foremost above the glass transition temperature of PA 6. Furthermore, they observed that crystallization behaviour of PA 6 gets affected by HNT's and good turn formation of g-modification for the low molar mass PA 6. They recommended that the effective arrangement of crystalline phase in presence of HNT's causes the reinforcement effect at low filler fraction. The flow properties also fairly changed by the addition of HNT's. Even, no any sign of a filler network was observed at $30 \%$

\section{Volume 4 Issue 12, December 2015}




\section{International Journal of Science and Research (IJSR) \\ ISSN (Online): 2319-7064}

Index Copernicus Value (2013): 6.14 | Impact Factor (2014): 5.611

filler concentration. Hence PA 6/halloysite composites can be still processed under similar conditions as neat polyamide 6 . In summary, HNT's develop the elastic properties of PA 6 without loosing flow properties of PA 6. Consequently, halloysite is an appealing alternative to the wollastonite and kaolin. By sufficient selection of HNT's with high aspect ratio, composites with higher potentials for the applications can be achieved.

D.C.O. Marney and Russell [18] presented the fire behaviour of HNT-nylon 6 composites. They found that halloysite nanotubes influence the fire performance of the composites, they developed a thermal insulation one kind of barrier on the surface of the composite at the time of burning. This barrier retards the burning without stopping it and more than doubled the total burning time. In general, it is similar to the fire behaviour of analogous nanoclay composites, however, much more HNT' were required to achieve a similar performance. In their study fire behaviour in terms of the smoke chemistry is not clear, so there is an opportunity to concentrate on evolution and characterization of evolved gases from the systems.

Mingliang Du et all. [3] prepared carboxylated butadienestyrene rubber (xSBR)/HNT nanocomposites with alone dispersed HNTs and strong interfacial bonding by the method known as co-coagulation. In the nanocomposites very strong hydrogen bond was prepared. The vulcanization of $\mathrm{xSBR} / \mathrm{HNT}$ compounds was belated with the addition of HNT's in small amount, and accelerated with more amount. Also they found the mechanical properties gets affected by addition of HNT's especially hardness and modulus.

Because of their tubular structure HNT's exhibits properties for getting better thermal stability and declining flammability of polypropylene. The temperature found to be $60{ }^{\circ} \mathrm{C}$ more than the neat PP at 5\% wt. loss in nitrogen of nanocomposite filled with $10 \mathrm{phr}$ modified HNT's, and was $74^{\circ} \mathrm{C}$ higher at maximum wt. loss. The flammability property of PP gets affected by HNTs. The thermal stability and flame retardant properties of HNTs on PP are because of hollow tubular structures of HNTs, the barriers for heat and mass transfer and the occurrence of iron in HNTs. Mingliang Du et all. [4] proved that HNTs is a new kind inexpensive, easily processed and effective additive for improving the thermal stability and flame retardant of PP.

\section{Conclusion}

The thermomechanical and physical properties are significantly enhanced by the incorporation of HNTs in different halloysite filled polymer blends and its composites.

\section{Effect of surface modification/treatment of HNT-}

HNTs speed up the phase separation process which gives rise to the formation of micro-papilla like hybrid spherulites with nanostructures on the composite surfaces, by playing the role of heterogeneous nuclei for PP. The surface having micro nano roughness are superhydrophobic in nature which has highest water contact angle more than $160^{\circ}$ as well as sliding angle less than $10^{\circ}$. The properties such as surface roughness, size of spherulites, and wetting property can be enhance by HNTs. The thermal degradation behaviour of PP can be appreciably enhanced by HNTs which is endorsed to the well-dispersed HNTs and the strong interfacial bonding by the nucleation. Mingxian Liu et all. [5] provided a substitute procedure for making the polymer superhydrophobic surfaces by modifying the microstructure of the surface just with the help of addition of nanoparticles in the melt. This process is also applicable for other polymers/nanoparticles systems in order to investigate its effectiveness.

The effect of loading ammonium salt modified halloysite nanotubes (QM-HNTs) and unmodified HNTs on mechanical, structural properties of PP were investigated by K. Prashantha et all. [24] for different HNTs wt. contents. They revealed that crystallization temperature as well as degree of crystallinity both increases with HNTs loading in $\mathrm{PP}$, thus showing an effect of potential nucleation induced the nanotubes. As compared to unmodified HNTs, QMHNTs shows good dispersion in PP matrix. They found a small increase in $\mathrm{Tg}$ and also enhancement in storage modulus with the inclusion of HNTs, more significant for modified than unmodified HNTs. They also, found that without considerable loss in ductility, modulus and strength of the nanocomposites increases with HNTs loading. The optimum value found for HNT wt. \% loading was 6 to achieve better properties of nanocomposites. QM-HNTs show better performance in terms of dispersion, mechanical properties than unmodified HNTs.

Virgin blend and the nanocomposites were prepared with the help of melt mixing method by Parthajit Pal et all. [23] they studied crystallinity and mechanical properties of these nanocomposites. They found HNTs acting as nucleating agent which increases the $\%$ crystallinity. Also, better dispersion is found after treating HNTs which remains in two phases, resulting enhanced performance.

Yubin $\mathrm{Fu}$ and Lide Zhang [19] suggested different metals or alloys such as $\mathrm{Co}, \mathrm{Fe}$ etc. that can be deposited on the halloysite template in order to prepare an interesting material for its low weight, low cost, and high magnetic property.

\section{Conclusion}

HNTs after surface treatment uniformly dispersed in blend matrices as compared to unmodified HNTs which ultimately improves the performance of nanocomposite. Especially the QM-HNTs gives better performances because of strong interfacial interaction between the polymer matrix and the nanotubes. Different metals and alloys such as Co, Fe etc. can also be deposited on halloysite template to produce an interesting material for its low weight, low cost, and high magnetic property.

\section{EPDM nanocomposites filled by Halloysite-}

Use H. Ismail et all. [12] reveal that dispersion of HNTs in the EPDM matrix was homogenous and three dimensional. At high HNT addition, they found interfacial and intertubular interactions among HNTs and EPDM, as well as 


\section{International Journal of Science and Research (IJSR) \\ ISSN (Online): 2319-7064}

Index Copernicus Value (2013): 6.14 | Impact Factor (2014): 5.611

face-to-edge and edge-to-edge interactions between HNTs i.e the zig-zag structure, because of which the simultaneous increases in tensile strength, stiffness and ductility of EPDM/HNT nanocomposites takes place. The better flame retardancy and thermal stability of the nanocomposites established because of the entrapment of degradation products of EPDM inside the lumen structure of tubules.

Pooria Pasbakhsh et all. [11] As compared to compatibilized EPDM/HNT nanocomposites the degree of intercalation of the HNTs is much more in an uncompatibilized nanocomposites. Also, the tensile property is because of the interphase generated in EPDM and HNT by MAH-g-EPDM which helps in making stronger interfacial interactions. On the other side, attendance of this compatibilizer bring down the curing time ( $\mathrm{t} 90$ ) but highers the maximum and minimum torques and also swelling resistance of the compatibilized EPDM/HNT nanocomposites compared to uncompatibilized EPDM/HNT nanocomposites. They also found that more HNT addition reduces the tensile strength because of the generation of two different phases of EPDM-rich and HNT rich areas.

\section{Conclusion}

The dispersion of HNTs in the EPDM matrix is homogenous and three dimensional. There is inter-tubular and interfacial interaction between HNTs and EPDM at high HNT addition, because of which the simultaneous increases in tensile strength, stiffness and ductility of EPDM/HNT nanocomposites takes place. The better flame retardancy and thermal stability of the nanocomposites established because of the entrapment of degradation products of EPDM inside the lumen structure of tubules.

\section{Dispersion of HNT's-}

Baochun Guo and Yanda Lei [10] used methacrylic acid to improve the performance of styrenebutadiene rubber/HNTs nanocomposites by direct blending. The ZDMA and MAAintermediated linkages result the strong interfacial bond among rubber matrix and HNTs. They found enhanced dispersion of HNTs in virtue of the interactions in-between HNTs and MAA or ZDMA. Also they obtain better mechanical properties of MAA-modified SBR/HNTs nanocomposites.

The accelerator (CBS) generally used in tire industry was used as compatibilizer for the PP/HNTs composites by Mingxian Liu and Baochun Guo [17]. CBS decompose at high temperature and yield benzothiazole sulphide radicals that act in response with the PP to yield benzothiazole sulfide grafted PP. On another side, the benzothiazoles on the grafted PP are reactive to HNTs using electron transfer. Consequently, the CBS works as a particular compatibilizer for the PP/HNTs composites. with adding up the CBS, high interfacial bonding and improved dispersion is possible. This shows a new interfacial design using interfacial grafting/electron transferring for the compatibilization of $\mathrm{PP} /$ clay composites.

B. Lecouvet and J.G. Gutierrez [2] reveal an improved dispersion and an alignment of the nanotubes in nanocomposites made by TSMC. Also, improvement in the storage modulus and the linear viscoelastic property. The entrapment of volatile products within the lumen structure of HNTs resulted an enhanced thermal stability of PA12/HNTs nanocomposites. Also they stated that halloysite has two effects on the non-isothermal crystallization behaviour of PA12, based on the HNTs content: improved nucleation and reduced mobility of the polyamide chains. The effect of nucleation is more prominent for twin-screw compounded nanocomposites with well-dispersed HNTs.

\section{Conclusion}

MAA can be used to modify SBR/HNTs composites in order to get good dispersion of HNTs and to obtain strong bond. The interactions among MAA and HNTs or ZDMA considerably enhances mechanical properties as well as dispersion of HNTs. Also with the addition of CBS, improved dispersion of HNTs and high interfacial bonding can be achieved.

\section{Modification of HNT's-}

V. Khunova et all. [25] indicated low impact of single halloysite modifications via ionic exchange, silane condensation and urea intercalation on parameters of $\mathrm{PP} /$ nanocomposites. The most significant effects of the modifiers practical consist of the effect on matrix crystallinity, especifically in relation with the HEDA and urea. However, the mechanical properties enhances by continuous loading of DBMI to PP/HNT due to the in situ modification of matrix, also increases the polarity of matrix and hence the affinity of the PP for the HNT. This combination results in better mechanical properties because of the combination between HNT and PP by helping the reaction between the $\mathrm{OH}$ groups of HNT and the imide ring of the DBMI by urea.

Baochun Guo et all. [7] Used Sorbic acid to get better results of styrenebutadiene rubber/halloysite nanotubes nanocomposites by direct blending. The SA intermediated linkages impart strong bonding between rubber matrix and the HNTs. Better dispersion of HNTs in virtue of the interactions between HNTs and SA were achieved by authors. Very good mechanical properties can be achieved from styrenebutadiene rubber/halloysite nanotubes nanocomposites.

Natural nanocomposites can be produced based on natural HNTs and natural rubber. A silane bonding media can be effectively utilized to improve the rubber filler chemical interactions. Sandip Rooj et all. [15] put forwarded their work with the preparation of NR-silica composites which was tailored with the same organosilane for comparative study between them. However, the limited intercalation of HNTs by the NR matrix was observed by them. Also they found better dispersion of HNTs in the matrix. But, magnificent enhancement of thermal stability for HNTs filled natural rubber.

Xiumei Sun et all. [13] developed a new HNT/chitosan 


\section{International Journal of Science and Research (IJSR) \\ ISSN (Online): 2319-7064 \\ Index Copernicus Value (2013): 6.14 | Impact Factor (2014): 5.611}

composite film to employ for the immobilization of horseradish peroxidase. Author stated that the HNTs can be successfully adhered on GC electrode by means of chitosan as a binder to form the composite film. The film provides a suitable matrix to help the direct electrochemistry of HRP and catalyze the reduction of $\mathrm{H} 2 \mathrm{O} 2$. This film gives a new hopeful platform for further study of the direct electrochemistry of redox proteins and the improvement of third-generation biosensors.

\section{Conclusion}

The most significant effects of the modifiers practical consist of the effect on matrix crystallinity, especifically in relation with the HEDA and urea. However, the mechanical properties enhances by continuous loading of DBMI to $\mathrm{PP} / \mathrm{HNT}$ due to the in situ modification of matrix, also increases the polarity of matrix and hence the affinity of the PP for the HNT. SA intermediated linkages impart strong bonding between rubber matrix and the HNTs. Better dispersion of HNTs in virtue of the interactions between HNTs and SA can be achieved. Also, very good mechanical properties can be achieved from styrenebutadiene rubber/halloysite nanotubes nanocomposites. A silane bonding media can be effectively utilized to improve the rubber filler chemical interactions. HNTs can be successfully adhered on GC electrode by means of chitosan as a binder to form the composite film.

\section{Nanocomposites based on Silicate Nanotubes-}

Katrin et all. [20] processed, injection moulded samples of nanocomposites consisting 2 and $5 \mathrm{wt} \%$ halloysite and were compared to those of nanocomposites based on organically modified clay. They exposed the potential of the HNTs as a reinforcing substance in polymers. Beside an improvement of elastic modulus and tensile strength of the polyamide- 6 matrix by the addition of halloysite, the toughening effect of the silicate nanotubes particularly must be emphasized. This predestines the HNT's for usage, which insist a consistent enhancement of toughness and strength.

\section{Conclusion}

An enlarged stiffness and strength as well as an improved elongation at break at little halloysite content can be obtained by mixing the silicate nanotubes of mineral halloysite in polymers. Nanocomposites based on silicate nanotubes provides better mechanical properties in terms of tensile strength and storage modulus. There lies a great opportunity to study and enhance the properties of HNT's by proper chemical action.

\section{Morphology and Crystallization Behavior}

Nan-ying Ning et all. [6] found uniform combination of HNTs in PP matrix for every samples prepared with varying content, and acts as a nucleation agent, showing an improvement of the whole crystallization rate. On the other side, they observed that at given isothermal crystallization temperature there is constant growth of spherulite, and suggested that spherulite expansion and nucleation are two independent methods. With 10 wt. \% HNT's loading little development of impact as well as tesile strength of PP/HNT composites was observed, it was because of constant crystallinity, unaffected size of spherulite of PP and less length to diameter ratio of the HNT's.

Non-isothermal crystallization and the polymorphism of the PA6 and the PA6/HNTs nanocomposites have been studied by Baochun Guo et all. [21] with the help of XRD analysis, DSC analysis and POM observations and Hoffman-Lauritzen nucleation theory. Because of the powerful interaction among HNT's and PA6 chains, HNT's performs as nucleating agent as well as decreases mobility of the PA6 chains. Also, HNTs speed up the crystallization in the concentration range. They also found with the help of kinetics analysis that the fold surface free energy of PA6/HNTs nanocomposites is bigger and was constrained at more HNT addition. It was also observed by them that the crystallinity of the PA6/HNTs nanocomposites highers with cooling rate. Crystallinity was found to be mostly affected by HNT's content.

Mingxian Liu et all. [9] halloysite nanotubes are known to have dual nucleating capacity for $\alpha$ and $\beta$ iPP under an isothermal and non-isothermal crystallization. The HNT's loading affects the generation of $\beta$-iPP in the iPP/HNTs composites. The maximum amount of $\beta$-iPP was found in composite with $20 \mathrm{phr}$ of HNTs. The amount of $\beta$-iPP maximises with reduction in cooling rate under the non isothermal crystallization. At the cooling rate of $2.5^{\circ} \mathrm{C} / \mathrm{min}$, more amount of $\beta$-crystal content was found. The structure of the $\beta$-iPP can be recognized as $\beta$-hedrites with axialite and flower cup like formation. The $\beta$-crystal may be produced between 115 to $140{ }^{\circ} \mathrm{C}$ in an isothermal crystallization, out of this range $\beta$-iPP cannot be found, it reaches at most to 135 ${ }^{\circ} \mathrm{C}$.

\section{Conclusion}

The HNT's get equally dispersed in the PP matrix, and acts as a nucleation mediator, which imparts improvement of the overall crystallization rate. The Higher the amount of HNTs, larger is the $\%$ of $\gamma$-phase crystals take up.

\section{Environmentally friendly HNT's-}

Environmentally friendly HNTs were introduced by K. Prashantha et all. [22]. They mixed natural nanotubes into various thermoplastics in order to produce new nanocomposite by the melt-mixing process. They found even distribution of HNT's in many polymers. The addition of natural nanotubes not only improves the mechanical properties of the naniocomposite but also increases its sustainability and environmental impact. They are also biocompatible, hence applied in medical fields.

\section{Conclusion}

The addition of natural nanotubes not only improves the mechanical properties of the naniocomposite but also increases its sustainability and environmental impact. They are also biocompatible, hence applied in medical fields. 


\section{International Journal of Science and Research (IJSR) \\ ISSN (Online): 2319-7064}

Index Copernicus Value (2013): 6.14 | Impact Factor (2014): 5.611

\section{Summary}

The study reveals that a lot work has been done on HNT filled polymer blends and its composites to improve the thermomechanical performance and physical properties. Researcher works to study the effect of HNT loading wt.\%, surface modification/treatment of HNT, EPDM nanocomposites filled by halloysite, dispersion of HNTs, effect of HNT modification, nanocomposites based on silicate nanotubes, morphology and crystallization behavior, Processing and characterization, environmentally friendly HNTs, has been employed for increase the thermomechanical and physical properties. There is lot of gap and no work has done on processing and characterization of halloysite (HNT's) filled polypropylene (PP)/ acrylonitrile butadiene styrene (ABS) blends and its composites to improve mechanical properties and thermal stability to establish the structure property relationship studies.

\section{References}

[1] H. Ismail, S.M. Shaari "Curing characteristics, tensile properties and morphology of palm ash/halloysite nanotubes/ethylene-propylene-diene monomer (EPDM) hybrid composites." Polymer Testing 29 (2010) 872-878 Elsevier Science Direct.

[2] B. Lecouvet, J.G. Gutierrez, M. Sclavons, C. Bailly "Structureproperty relationships in polyamide 12/halloysite nanotube nanocomposites." Polymer Degradation and Stability 96 (2011) 226e235 Elsevier Science Direct.

[3] Mingliang Du, Baochun Guo, Yanda Lei, Mingxian Liu, Demin Jia "Carboxylated butadiene-styrene rubber/halloysite nanotube nanocomposites: Interfacial interaction and performance" Polymer 49 (2008) 48714876 Elsevier Science Direct.B. Smith, "An approach to graphs of linear forms (Unpublished work style)," unpublished.

[4] Mingliang $\mathrm{Du}$, Baochun Guo, Demin Jia "Thermal stability and flame retardant effects of halloysite nanotubes on poly (propylene)." European Polymer Journal 42 (2006) 1362-1369 Elsevier Science Direct.

[5] Mingxian Liu a, b, Zhixin Jia a, Fang Liu a, Demin Jia a, Baochun Guo "Tailoring the wettability of polypropylene surfaces with halloysite nanotubes." Journal of Colloid and Interface Science 350 (2010) 186-193 Elsevier Science Direct.

[6] Nan-ying Ning, Qin-jian Yin, Feng Luo, Qin Zhang, Rongni $\mathrm{Du}$, Qiang $\mathrm{Fu}$ "Crystallization behavior and mechanical properties of polypropylene/halloysite composites" Polymer 48 (2007) 7374e 7384 Science Direct M. Young, The Techincal Writers Handbook. Mill Valley, CA: University Science, 1989.

[7] Baochun Guo, Feng Chen, Yanda Lei, Xiaoliang Liu, Jingjing Wan, Demin Jia "Styrene-butadiene rubber/halloysite nanotubes nanocomposites modified by sorbic acid" Applied Surface Science 255 (2009) 7329-7336

[8] Chao Yang, Peng Liu, Yongqing Zhao "Preparation and characterization of coaxial halloysite/polypyrrole tubular nanocomposites for electrochemical energy storage." Electrochimica Acta 55 (2010) 6857-6864.

[9] Liu, Baochun Guo, Mingliang Du, Feng Chen, Demin Jia "Halloysite nanotubes as a novel $\beta$-nucleating agent for isotactic polypropylene." Mingxian Polymer 50 (2009) 3022-3030

[10] Baochun Guo, Yanda Lei, Feng Chen, Xiaoliang Liu, Mingliang Du, Demin Jia "Styrene-butadiene rubber/halloysite nanotubes nanocomposites modified by methacrylic acid."Applied Surface Science 255 (2008) 2715-2722

[11] Pooria Pasbakhsh, H. Ismail, M.N. Ahmad Fauzi, A. $\mathrm{Abu}$ Bakar "Influence of maleic anhydride grafted ethylene propylene diene monomer (MAH-g-EPDM) on the properties of EPDM nanocomposites reinforced by halloysite nanotubes." Polymer Testing 28 (2009) 548559

[12] H. Ismail*, Pooria Pasbakhsh, M.N. Ahmad Fauzi, A. Abu Bakar "Morphological, thermal and tensile properties of halloysite nanotubes filled ethylene propylene diene monomer (EPDM) nanocomposites." Polymer Testing 27 (2008) 841-850

[13] Xiumei Sun, HebaiShen, NengqinJia "Direct electrochemistry and electrocatalysis of horseradish peroxidase based on halloysite nanotubes/chitosan nanocomposite film."Electrochimica Acta 56 (2010) 700-705 Electrochimica Acta 56 (2010) 700-705

[14] Ulrich A. Handge a, Katrin Hedicke-Höchstötter b, Volker Altstädt "Composites of polyamide 6 and silicate nanotubes of the mineral halloysite: Influence of molecular weight on thermal, mechanical and rheological properties." Polymer 51 (2010) 2690e2699

[15] Sandip Rooj a, Amit Das a, Varun Thakur a, R.N. Mahaling a, Anil K. Bhowmick b, Gert Heinrich a, "Preparation and properties of natural nanocomposites based on natural rubber and naturally occurring halloysite nanotubes." Materials and Design 31 (2010) 2151-2156

[16] Yanfang Xiea, Peter R. Changb, Shujun Wangc, Jiugao Yua, Xiaofei Ma "Preparation and properties of halloysite nanotubes/plasticized Dioscorea opposite Thunb. starch composites." Carbohydrate Polymers 83 (2011) 186-191

[17] Mingxian Liu, Baochun Guo, Yanda Lei, Mingliang Du, Demin Jia "Benzothiazole sulfide compatibilized polypropylene/halloysite nanotubes composites" Applied Surface Science 255 (2009) 4961-4969

[18] D.C.O. Marney a, L.J. Russell a, D.Y. Wu a, T. Nguyen a, D. Crammb, N. Rigopoulos a, N. Wright a, M. Greaves c "The suitability of halloysite nanotubes as a fire retardant for nylon 6." Polymer Degradation and Stability 93 (2008) 1971-1978

[19] Yubin Fu a, b, Lide Zhang a "Simultaneous deposition of $\mathrm{Ni}$ nanoparticles and wires on a tubular halloysite template: A novel metallized ceramic microstructure." Journal of Solid State Chemistry 178 (2005) 3595-3600

[20] Katrin Hedicke-Höchstötter a, Goy Teck Lim b, Volker Altstädt c "Novel polyamide nanocomposites based on silicate nanotubes of the mineral halloysite" Composites Science and Technology 69 (2009) 330-334

[21] Baochun Guo, Quanliang Zou, Yanda Lei, Mingliang Du, Mingxian Liu, Demin Jia "Crystallization behavior 
of polyamide $6 /$ halloysite nanotubes nanocomposites." Thermochimica Acta 484 (2009) 48-56

[22] K. Prashantha, M.-F.Lacrampe, P.Krawczak "Halloysite Nanotubes-Polymer Nano composites: A New Class of Multifaceted Materials" Advanced Materials Manufacturing \& Characterization Vol3 Issue 1 (2013)

[23] "Mechanical and Crystalline Behavior of Polymeric Nanocomposites in Presence of Natural Clay" Parthajit Pal1, Mrinal Kanti Kundu1, Swinderjeetsingh Kalra2, Chapal Kumar Das1 Open Journal of Applied Sciences, 2012, 2, 277-282

[24] K. Prashantha, M. F. Lacrampe, P. Krawczak "Processing and characterization of halloysite nanotubes filled polypropylene nanocomposites based on a masterbatch route: effect of halloysites treatment on structural and mechanical properties" express Polymer Letters Vol.5, No.4 (2011) 295-307

[25] V. Khunova1, J. Kristof2, I. Kelnar3, J. Dybal3 “The effect of halloysite modification combined with in situ matrix modifications on the structure and properties of polypropylene/halloysite nanocomposites" eXPRESS Polymer Letters Vol.7, No.5 (2013) 471-479

\section{Author Profile}

Pravin R. Kubade received the M.E. degree in MechanicalProduction Engineering from Government College of Engineering Karad- Maharashtra, INDIA., and pursuing Ph.D. in Mechanical Engineering from SMBS, VIT University, Vellore-T.N. , INDIA. 